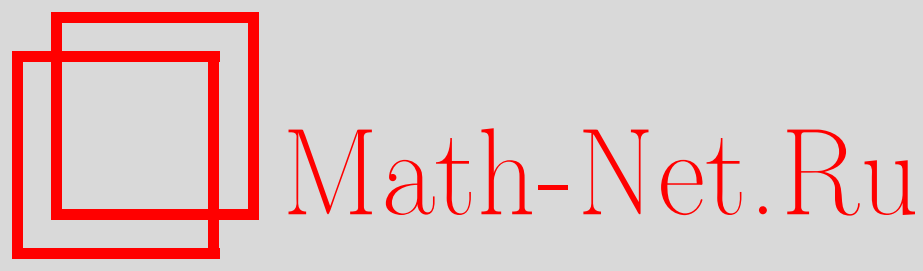

Я. Мозер, Лестницы Иакова, факторизация и метаморфозы в качестве приложения к функциональному уравнению Римана для $\zeta(s)$ на критической прямой, Совр. пробл. матем., 2016, выпуск 23, 102-113

DOI: https://doi.org/10.4213/spm65

Использование Общероссийского математического портала Math-Net.Ru подразумевает, что вы прочитали и согласны с пользовательским соглашением http://www.mathnet.ru/rus/agreement

Параметры загрузки:

IP: 18.208 .226 .222

26 апреля 2023 г., 11:10:26

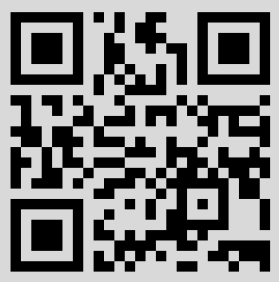




\title{
Лестницы Иакова, факторизация и метаморфозы в качестве приложения к функциональному уравнению Римана для $\zeta(s)$ на критической прямой
}

\author{
Я. Мозер \\ Comenius University, Slovakia \\ jan.mozer@fmph.uniba.sk
}

Посвящается памяти Анатолия Алексеевича Карачубъ

\section{1. Введение и первый результат}

1.1. Напомним функциональное уравнение Римана (1859 г.):

$$
\zeta(1-s)=2(2 \pi)^{-s} \cos \frac{\pi s}{2} \Gamma(s) \zeta(s), \quad s \in \mathbb{C} \backslash\{1\} .
$$

ЗАмечАниЕ 1. Известно, что Л. Эйлер открыл формулу, эквивалентную (1) для вещественных значений переменной $s$ в 1749 г. (см. [1; с. 23-26]), однако доказательство в работе Эйлера отсутствует.

Теперь если мы положим

$$
\chi(s)=\pi^{s-1 / 2} \frac{\Gamma(1 / 2-s / 2)}{\Gamma(s / 2)} \quad \Longrightarrow \quad \chi(s) \chi(1-s)=1
$$

(см. $[2 ;$ с. 13,16$])$, то получим, что

$$
\frac{\zeta(1-s)}{\zeta(s)}=\chi(s)
$$

ЗАмЕчаниЕ 2. В связи с (3) используем следующее (Э. Ландау, [3; с. 30]): отношение

$$
\frac{\zeta(1-s)}{\zeta(s)}
$$

выражается через известную функцию ... (ясно, что эта известная функция отличается от $\zeta(s))$.

1.2. Однако в случае

$$
s=\frac{1}{2}+i t, \quad 1-s=\frac{1}{2}-i t=\left(\frac{1}{2}+i t\right)^{\star}
$$

имеем (из (2), (4)), что

$$
\left|\chi\left(\frac{1}{2}+i t\right)\right|=1
$$

т.е. (см. (3))

$$
\left|\overline{\frac{\zeta(1 / 2+i t)}{\zeta(1 / 2+i t)}}\right|=1 \quad \text { для всех } \quad t \neq \gamma: \quad \zeta\left(\frac{1}{2}+i \gamma\right)=0
$$

(вслед за продолжением на $t=\gamma$ это справедливо для всех $t \in \mathbb{R}$ ). 
ЗАмЕчАНИЕ 3. На критической прямой

$$
s=\frac{1}{2}+i t
$$

Риманово функциональное уравнение (3) дает только тривиальный результат (5) (ясно, что главная цель функционального уравнения Римана - это аналитическое продолжение $\zeta(s)$ на $\mathbb{C} \backslash\{1\})$.

1.3. Наше замечание 2, очевидно, приводит к следующему вопросу.

Вопрос. Как иначе выбрать точки

$$
t_{2}>t_{1}>0
$$

(скажем) из соответствующего множества, чтобы отношение (из (3), (5))

$$
\left|\frac{\zeta\left(1 / 2+i t_{2}\right)}{\zeta\left(1 / 2+i t_{1}\right)}\right|, \quad t_{1}, t_{2} \neq \gamma
$$

выражалось известной функцией?

Существует способ дать ответ на вопрос, причем один ответ из набора многих возможных - это наш метод преобразования с помощью интегралов, взятых в противоположных направлениях (см. [4; (4.1)-(4.19)]).

1.4. В настоящей работе мы получаем, например, следующее.

ФормУлА 1. Для любого достаточно большого $L \in \mathbb{N}$ и любого $U \in(0, \pi)$ существуют функции

$$
\begin{gathered}
\alpha_{0}^{4}=\alpha_{0}^{4}(L, U ; a, b), \quad \alpha_{1}^{4}=\alpha_{1}^{4}(L, U ; a, b), \quad \beta_{1}^{4}=\beta_{1}^{4}(L, U), \\
\alpha_{0}^{4}, \alpha_{1}^{4}, \beta_{1}^{4} \neq \gamma: \quad \zeta\left(\frac{1}{2}+i \gamma\right)=0
\end{gathered}
$$

такие, что

$$
\left|\frac{\zeta\left(1 / 2+i \alpha_{1}^{4}\right)}{\zeta\left(1 / 2+i \beta_{1}^{4}\right)}\right| \sim \frac{\operatorname{arctg}(\sqrt{(a-b) /(a+b)} \operatorname{tg}(U / 2))}{\sqrt{(a-b) /(a+b)}(U / 2)} \frac{a+b \cos \left(\alpha_{0}^{4}\right)}{a+b}, \quad L \rightarrow \infty
$$

где

$$
\begin{gathered}
\alpha_{0}^{4} \in(2 \pi L, 2 \pi L+U), \quad \alpha_{1}^{4}, \beta_{1}^{4} \in\left(\stackrel{1}{2 \pi L}, 2 \pi L^{1}+U\right), \\
(2 \pi L, 2 \pi L+U) \prec\left(2 \pi L, 2 \pi L^{1}+U\right),
\end{gathered}
$$

и функции (8) обладают следующим свойством:

$$
\alpha_{1}^{4}-\alpha_{0}^{4} \sim(1-c) \boldsymbol{\pi}(2 \pi L), \quad L \rightarrow \infty,
$$

где $c$ - постоянная Эйлера и $\boldsymbol{\pi}(t)$ - функция распределения простых чисел.

ЗАмечАние 4. Формула (9) дает ответ на вопрос (из (7), (10)) в направлении, указанном в замечании 2 .

ЗАмечание 5. Отметим, что в явном виде в случае (7), т.е. на критической прямой, мы можем предположить, что известная функция - это любая функция, например,

$$
f[|\zeta|], \quad \arg \zeta, \quad \ldots .
$$


В этом направлении мы получили следующее (см. также соответствующие формулы в статьях [4]-[6], $k=1$ )

ФормУЛА 2. Имеем

$$
\left|\frac{\zeta\left(1 / 2+i \alpha_{1}^{2}\right)}{\zeta\left(1 / 2+i \beta_{1}^{2}\right)}\right| \sim \frac{\sqrt{\zeta(2 \sigma)}}{\left|\zeta\left[\sigma+i \alpha_{0}^{2}(\sigma)\right]\right|}, \quad \sigma \geqslant 1 .
$$

ФормУлА 3. Имеем

$$
\left|\frac{\zeta\left(1 / 2+i \alpha_{1}^{3}\right)}{\zeta\left(1 / 2+i \beta_{1}^{3}\right)}\right| \sim \pi^{l} \sqrt{c_{l}}\left|\int_{0}^{\alpha_{0}^{3}(T)} \arg \zeta\left(\frac{1}{2}+i t\right) d t\right|^{-l} .
$$

ФормУлА 4. Имеем

$$
\left|\frac{\zeta\left(1 / 2+i \alpha_{1}^{1}\right)}{\zeta\left(1 / 2+i \beta_{1}^{1}\right)}\right| \sim \sqrt[4]{\frac{2 \pi}{H}} \frac{1}{\sqrt{\left|\zeta\left(1 / 2+i \alpha_{0}^{1}\right)\right|}} .
$$

ЗАмечание 6. Результаты (9), (11)-(13) основаны на таких свойствах лестницы Иакова $\varphi_{1}(t):$

$$
\begin{gathered}
\alpha_{0}^{4}=\varphi_{1}^{1}(d)=\varphi_{1}(d) \in(2 \pi L, 2 \pi L+U), \quad \alpha_{1}^{4}=\varphi_{1}^{0}(d)=d \in\left(2 \pi L, 2 \pi L^{1}+U\right), \\
\beta_{1}^{4}=\varphi_{1}^{0}(e)=e \in\left(2 \pi L, 2 \pi L^{1}+U\right) .
\end{gathered}
$$

1.5. Напомним для полноты картины, что лестница Иакова

$$
\varphi_{1}(t)=\frac{1}{2} \varphi(t)
$$

была введена в нашей работе [7] (см. также [8]), где функция $\varphi(t)$ - произвольное решение нелинейного интегрального уравнения

$$
\int_{0}^{\mu[x(T)]} Z^{2}(t) e^{-t(2 / x(T))} d t=\int_{0}^{T} Z^{2}(t) d t,
$$

в котором каждая допустимая функция $\mu(y)$ порождает решение

$$
y=\varphi(T ; \mu)=\varphi(T), \quad \mu(y) \geqslant 7 y \ln y .
$$

Функция $\varphi_{1}(t)$ называется лестницей Иакова в соответствии со "сном Иакова в Хумаше, Берейшит, 28:12".

ЗАмЕчАНИЕ 7. Применением лестниц Иакова показано (см. [7]), что классический интеграл Харди-Литтлвуда (1918)

$$
\int_{0}^{T}\left|\zeta\left(\frac{1}{2}+i t\right)\right|^{2} d t
$$

выражается - в дополнение к формуле Харди-Литтлвуда (и другим аналогичным), обладающей неограниченной ошибкой при $T \rightarrow \infty,-$ следующим бесконечным множеством почти точных формул:

$$
\int_{0}^{T}\left|\zeta\left(\frac{1}{2}+i t\right)\right|^{2} d t=\varphi_{1}(T) \ln \varphi_{1}(T)+(c-\ln 2 \pi) \varphi_{1}(T)+c_{0}+\mathcal{O}\left(\frac{\ln T}{T}\right), \quad T \rightarrow \infty
$$

где $c$ - константа Эйлера и $c_{0}$ - константа из формулы Тичмарша-Кобера-Аткинсона. 
ЗАмечАниЕ 8. Лестница Иакова $\varphi_{1}(t)$ может быть истолкована из нашей формулы (cM. [7])

$$
T-\varphi_{1}(T) \sim(1-c) \pi(T), \quad \pi(T) \sim \frac{T}{\ln T}
$$

где $\pi(T)$ является функцией распределения простых чисел, как функция, асимптотически дополнительная к $(1-c) \pi(T)$ в следующем смысле

$$
\varphi_{1}(T)+(1-c) \pi(T) \sim T, \quad T \rightarrow \infty .
$$

\section{2. Факторизация, осциллирующая $Q$-система и ее метаморфозы в качестве общего функционального дополнения к уравнению Римана на критической прямой}

2.1. Осциллирующая $Q$-система определена в работе $[4 ;(2.1)]$ следующим образом:

$$
\begin{aligned}
G\left(x_{1}, \ldots, x_{k} ; y_{1}, \ldots, y_{k}\right) & \stackrel{\text { def }}{=} \prod_{r=1}^{k}\left|\frac{\zeta\left(1 / 2+i x_{r}\right)}{\zeta\left(1 / 2+i y_{r}\right)}\right| \\
& =\prod_{r=1}^{k}\left|\frac{\sum_{n \leqslant \tau\left(x_{r}\right)}(2 / \sqrt{n}) \cos \left\{\vartheta\left(x_{r}\right)-x_{r} \ln n\right\}+R\left(x_{r}\right)}{\sum_{n \leqslant \tau\left(y_{r}\right)}(2 / \sqrt{n}) \cos \left\{\vartheta\left(y_{r}\right)-y_{r} \ln n\right\}+R\left(y_{r}\right)}\right|, \\
\tau(t)=\sqrt{\frac{t}{2 \pi}}, \quad R(t)=\mathcal{O}\left(t^{-1 / 4}\right), \quad k \leqslant k_{0} \in \mathbb{N}, &
\end{aligned}
$$

для соответствующих множеств (см. [4; (2.2)]) точек

$$
\left(x_{1}, \ldots, x_{k}\right), \quad\left(y_{1}, \ldots, y_{k}\right) .
$$

ЗАмЕчАниЕ 9. Очевидно, что определение (15) основано на простом обобщении

$$
\left|\frac{\zeta(1 / 2+i x)}{\zeta(1 / 2+i y)}\right| \rightarrow \prod_{r=1}^{k}\left|\frac{\zeta\left(1 / 2+i x_{r}\right)}{\zeta\left(1 / 2+i y_{r}\right)}\right|
$$

(cм. (7), (11)-(13)).

Напомним некоторые из известных результатов, играющих роль функционального уравнения Римана на критической прямой.

(А) Существуют функции (см. [4; (2.5)])

$$
\begin{gathered}
\alpha_{r}^{2}=\alpha_{r}^{2}(\sigma, T, \Theta, k, \epsilon), \quad r=0,1, \ldots, k, \quad \beta_{r}^{2}=\beta_{r}^{2}(T, \Theta, k), \quad r=1, \ldots, k, \\
\alpha_{r}^{2}, \beta_{r}^{2} \neq \gamma: \quad \zeta\left(\frac{1}{2}+i \gamma\right)=0,
\end{gathered}
$$

для допустимых

$$
\sigma, T, \Theta, k, \epsilon
$$

такие, что справедлива следующая формула факторизации:

$$
\prod_{r=1}^{k}\left|\frac{\zeta\left(1 / 2+i \alpha_{r}^{2}\right)}{\zeta\left(1 / 2+i \beta_{r}^{2}\right)}\right| \sim \frac{\sqrt{\zeta(2 \sigma)}}{\left|\zeta\left[\sigma+i \alpha_{0}^{2}(\sigma)\right]\right|}, \quad T \rightarrow \infty
$$


(см. $[4 ;(2.6)])$, т.е. существует множество метаморфоз осциллирующей $Q$-системы $(15)$

$$
\begin{aligned}
& \prod_{r=1}^{k}\left|\frac{\sum_{n \leqslant \tau\left(\alpha_{r}^{2}\right)}(2 / \sqrt{n}) \cos \left\{\vartheta\left(\alpha_{r}^{2}\right)-\alpha_{r}^{2} \ln n\right\}+R\left(\alpha_{r}^{2}\right)}{\sum_{n \leqslant \tau\left(\beta_{r}^{2}\right)}(2 / \sqrt{n}) \cos \left\{\vartheta\left(\beta_{r}^{2}\right)-\beta_{r}^{2} \ln n\right\}+R\left(\beta_{r}^{2}\right)}\right| \\
& \quad \sim \sqrt{\zeta(2 \sigma)}\left|\sum_{n=1}^{\infty} \frac{\mu(n)}{\sigma+i \alpha_{0}^{2}(\sigma)}\right|, \quad \sigma>1+\epsilon, \quad T \rightarrow \infty
\end{aligned}
$$

(см. $[4 ;(2.6)])$, где $\mu(n)$ - функция Мёбиуса.

(B) Существуют функции

$$
\begin{gathered}
\alpha_{r}^{3}=\alpha_{r}^{3}(T, l, \epsilon, k), \quad r=0,1, \ldots, k, \quad l \in \mathbb{N}, \quad \beta_{r}^{3}=\beta_{r}^{3}(T, \epsilon, k), \quad r=1, \ldots, k, \\
\alpha_{r}^{3}, \beta_{r}^{3} \neq \gamma: \quad \zeta\left(\frac{1}{2}+i \gamma\right)=0,
\end{gathered}
$$

для допустимых

$$
T, l, \epsilon, k
$$

такие, что справедлива следующая формула факторизации:

$$
\left|\int_{0}^{\alpha_{0}^{3}} \arg \zeta(1 / 2+i t) d t\right| \sim \pi c^{1 /(2 l)} \prod_{r=1}^{k}\left|\frac{\zeta\left(1 / 2+i \alpha_{r}^{3}\right)}{\zeta\left(1 / 2+i \beta_{r}^{3}\right)}\right|^{-1 / l}, \quad T \rightarrow \infty
$$

(см. $[6 ;(2.4)])$, т.е. существует множество метаморфоз осциллирующей $Q$-системы $(15)$

$$
\begin{gathered}
\prod_{r=1}^{k}\left|\frac{\sum_{n \leqslant \tau\left(\alpha_{r}^{3}\right)}(2 / \sqrt{n}) \cos \left\{\vartheta\left(\alpha_{r}^{3}\right)-\alpha_{r}^{3} \ln n\right\}+R\left(\alpha_{r}^{3}\right)}{\sum_{n \leqslant \tau\left(\beta_{r}^{3}\right)}(2 / \sqrt{n}) \cos \left\{\vartheta\left(\beta_{r}^{3}\right)-\beta_{r}^{3} \ln n\right\}+R\left(\beta_{r}^{3}\right)}\right| \\
\sim \pi^{l} \sqrt{c_{l}}\left|\int_{0}^{\alpha_{0}^{3}} \arg \zeta\left(\frac{1}{2}+i t\right) d t\right|, \quad T \rightarrow \infty
\end{gathered}
$$

(см. $[6 ;(4.6)])$.

(B1) Если переписать формулу (18) следующим образом:

$$
\left|\int_{\mu_{m}}^{\alpha_{0}^{3}} \arg \zeta\left(\frac{1}{2}+i t\right) d t\right| \sim \pi c_{l}^{1 /(2 l)} \prod_{r 1=1}^{k}\left|\frac{\sum_{n \leqslant \tau\left(\alpha_{r}^{3}\right)}(2 / \sqrt{n}) \cos \left\{\vartheta\left(\alpha_{r}^{3}\right)-\alpha_{r}^{3} \ln n\right\}+R\left(\alpha_{r}^{3}\right)}{\sum_{n \leqslant \tau\left(\beta_{r}^{3}\right)}(2 / \sqrt{n}) \cos \left\{\vartheta\left(\beta_{r}^{3}\right)-\beta_{r}^{3} \ln n\right\}+R\left(\beta_{r}^{3}\right)}\right|^{-1 / l},
$$

где

$$
m=m\left(\alpha_{0}^{3}\right), \quad \mu_{m}<\alpha_{0}^{3}<\mu_{m+1}, \quad S_{1}\left(\mu_{m}\right)=0
$$

(см. [6; (4.11)]), то мы получим множество метаморфоз (19) в обратном направлении. А именно, мы начинаем с

$$
\left|\int_{0}^{w} \arg \zeta\left(\frac{1}{2}+i t\right) d t\right|
$$

т.е. с группы Аарона, скажем,

$$
\rightarrow\left|\int_{\mu_{m}}^{\alpha_{0}^{3}} \arg \zeta\left(\frac{1}{2}+i t\right) d t\right|
$$


т.е. зародыш группы Аарона (соответствующий $w=\alpha_{0}^{3}$ )

$$
\sim\left|\frac{\sum_{n \leqslant \tau\left(\alpha_{r}^{3}\right)}(2 / \sqrt{n}) \cos \left\{\vartheta\left(\alpha_{r}^{3}\right)-\alpha_{r}^{3} \ln n\right\}+R\left(\alpha_{r}^{3}\right)}{\sum_{n \leqslant \tau\left(\beta_{r}^{3}\right)}(2 / \sqrt{n}) \cos \left\{\vartheta\left(\beta_{r}^{3}\right)-\beta_{r}^{3} \ln n\right\}+R\left(\beta_{r}^{3}\right)}\right|
$$

уже преобразился “в миндалях созревших" (см. "Тора, 4-й книге Моисея, Хумаше, Бемидбар, $17: 23 ")$.

(C) Мы получили первый набор метаморфоз первозданной мультиформы

$$
G\left(x_{1}, \ldots, x_{k}\right)=\prod_{r=1}^{k}\left|\zeta\left(\frac{1}{2}+i x_{r}\right)\right|
$$

в статье [5]. Соответствующие результаты, выраженные в терминах осциллирующей $Q$-системы (15), это (см. [5; (1.7), (2.5)]) формула факторизации

$$
\prod_{r=1}^{k}\left|\frac{\zeta(1 / 2+i x)}{\zeta(1 / 2+i y)}\right| \sim \sqrt[4]{\frac{2 \pi}{H}} \frac{1}{\left|\zeta\left(1 / 2+i \alpha_{0}^{1}\right)\right|}
$$

и соответствующее множество метаморфоз осциллирующей $Q$-системы

$$
\begin{aligned}
& \prod_{r=1}^{k}\left|\frac{\sum_{n \leqslant \tau\left(\alpha_{r}^{1}\right)}(2 / \sqrt{n}) \cos \left\{\vartheta\left(\alpha_{r}^{1}\right)-\alpha_{r}^{1} \ln n\right\}+R\left(\alpha_{r}^{1}\right)}{\sum_{n \leqslant \tau\left(\beta_{r}^{1}\right)}(2 / \sqrt{n}) \cos \left\{\vartheta\left(\beta_{r}^{1}\right)-\beta_{r}^{1} \ln n\right\}+R\left(\beta_{r}^{1}\right)}\right| \\
& \quad \sim \frac{\sqrt[4]{2 \pi / H}}{\sqrt{\left|\sum_{n \leqslant \tau\left(\alpha_{0}^{1}\right)}(2 / \sqrt{n}) \cos \left\{\vartheta\left(\alpha_{0}^{1}\right)-\alpha_{0}^{1} \ln n\right\}+R\left(\alpha_{0}^{1}\right)\right|}}, \quad T \rightarrow \infty .
\end{aligned}
$$

(D) Кроме того, последовательности

$$
\left\{\alpha_{r}^{n}\right\}_{r=0}^{k}, \quad\left\{\beta_{r}^{n}\right\}_{r=1}^{k}, \quad n=1,2,3,
$$

обладают следующим свойством:

$$
\begin{aligned}
\alpha_{r+1}^{n}-\alpha_{r}^{n} & \sim(1-c) \pi(T), \quad r=0,1, \ldots, k-1, \\
\beta_{r+1}^{n}-\beta_{r}^{n} & \sim(1-c) \pi(T), \quad r=1, \ldots, k-1, \quad k \geqslant 2
\end{aligned}
$$

(см. (6), (7), замечания 8 и 9).

\section{3. Теорема; факторизация как аналог функционального уравнения Римана на критической прямой}

3.1. Воспользуемся следующим Эйлеровым интегралом (см. [9; с. 134-135]):

$$
\begin{aligned}
\int \frac{d \varphi}{a+b \cos \varphi} & =\frac{2}{\sqrt{a^{2}-b^{2}}} \operatorname{arctg} \frac{(a-b) \operatorname{tg}(\varphi / 2)}{\sqrt{a^{2}-b^{2}}}, \quad \varphi \in(0, \pi), \\
a+b & >0, \quad a^{2}-b^{2}>0 \quad \Longrightarrow \quad a>|b| .
\end{aligned}
$$

Из (22) сразу получаем следующую формулу:

$$
\int_{2 \pi L}^{2 \pi L+U} \frac{d \varphi}{a+b \cos \varphi}=\frac{1}{a+b} U \frac{\operatorname{arctg}(\sqrt{(a-b) /(a+b)} \operatorname{tg}(U / 2))}{\sqrt{(a-b) /(a+b)}(U / 2)}, \quad L \in \mathbb{Z}, \quad U \in(0, \pi) .
$$

Теперь если мы используем метод преобразования (см. [4; (4.1)-(4.19)]) в соответствии с формулой (23), то получим следующее утверждение. 
Teopema. Пусmъ

$$
\left.[2 \pi L, 2 \pi L+U] \rightarrow \stackrel{1}{\pi} L, \pi \stackrel{1}{\pi}+U], \ldots, \stackrel{k}{\pi} L, \pi \stackrel{k}{*} L^{+} U\right]
$$

где

$$
\left.\stackrel{r}{\pi L}, \pi L^{r}+U\right], \quad r=1, \ldots, k, \quad k \leqslant k_{0} \in \mathbb{N},
$$

есть сегменты, повторяющиеся в обратном направлении по отношению $к$ первому сегменту в (24), и $k_{0}$ есть произвольное фиксированное число. Тогда существует достаточно больwoe

$$
T_{0}=T_{0}(a, b)>0
$$

такое, что для каждого

$$
L>\frac{1}{2 \pi} T_{0}
$$

и любъх допустимых $L, U, k$ существуют функиии

$$
\begin{gathered}
\alpha_{r}^{4}(L, U, k ; a, b), \quad r=0,1, \ldots, k, \quad \beta_{r}^{4}(L, U, k), \quad r=1, \ldots, k, \\
\alpha_{r}^{4}, \beta_{r}^{4} \neq \gamma: \quad \zeta\left(\frac{1}{2}+i \gamma\right)=0,
\end{gathered}
$$

такие, что

$$
\prod_{r=1}^{k}\left|\frac{\zeta\left(1 / 2+i \alpha_{r}^{4}\right)}{\zeta\left(1 / 2+i \beta_{r}^{4}\right)}\right|^{2} \sim \frac{\operatorname{arctg}(\sqrt{(a-b) /(a+b)} \operatorname{tg}(U / 2))}{\sqrt{(a-b) /(a+b)}(U / 2)} \frac{a+b \cos \alpha_{0}^{4}}{a+b}, \quad L \rightarrow \infty
$$

причем

$$
G\left(\alpha^{4}, \beta^{4}\right) \sim \sqrt{(\ldots)} \quad \Longleftrightarrow \quad\left\{G\left(\alpha^{4}, \beta^{4}\right)\right\}^{2} \sim(\ldots) .
$$

Кроме того, последовательности

$$
\left\{\alpha_{r}^{4}\right\}_{r=0}^{k}, \quad\left\{\beta_{r}^{4}\right\}_{r=1}^{k}, \quad n=1,2,3,
$$

обладают следующими свойствами:

$$
\begin{gathered}
2 \pi L<\alpha_{0}^{4}<\alpha_{1}^{4}<\cdots<\alpha_{k}^{4}, \quad 2 \pi L<\beta_{1}^{4}<\beta_{2}^{4}<\cdots<\beta_{k}^{4}, \\
\alpha_{0}^{4} \in(2 \pi L, 2 \pi L+U), \\
{ }^{r}{ }_{r}^{4}, \beta_{r}^{4} \in\left(2 \pi L, 2 \pi L^{r}+U\right), \quad r=1,2, \ldots, k, \\
\alpha_{r+1}^{4}-\alpha_{r}^{4} \sim(1-c) \pi(2 \pi L), \quad r=0,1, \ldots, k-1, \\
\beta_{r+1}^{4}-\beta_{r}^{4} \sim(1-c) \pi(2 \pi L), \quad r=1, \ldots, k-1, \quad k \geqslant 2,
\end{gathered}
$$

¿əe

$$
\pi(T) \sim \frac{T}{\ln T}, \quad T \rightarrow \infty,
$$

- функция распределения простых чисел и с-константа Эйлера.

3.2. Теперь отметим следующее.

ЗАмЕчАниЕ 10. Асимптотическое поведение множеств

$$
\left\{\alpha_{r}^{4}\right\}_{r=0}^{k}
$$

следующее: если $L \rightarrow \infty$, то точки каждого множества (29) неограниченно разбегаются друг от друга и все вместе уходят на бесконечность; следовательно, при $L \rightarrow \infty$ каждое из множеств (29) ведет себя как одномерная вселенная Фридмана-Хаббла. 
ЗАмечание 11. Теперь представим результат (26), учитывая (1), (3), замечания 2 и 9, в виде

$$
\prod_{r=0}^{k}\left|\zeta\left(\frac{1}{2}+i \alpha_{r}^{4}\right)\right| \sim \sqrt{\chi_{4}\left(U, \alpha_{0}^{4}\right)} \prod_{r=1}^{k}\left|\zeta\left(\frac{1}{2}+i \beta_{r}^{4}\right)\right|
$$

где $\chi_{4}$ обозначает правую часть (26). Совершенно очевидно, что эта формула является аналогом функционального уравнения Римана на критической прямой.

ЗАмечАние 12. Ясно, что первый результат (9) - это особый случай (26).

\section{4. О множестве метаморфоз, которые соответствуют формуле (26)}

Напомним формулу Римана-Зигеля в спектральной форме:

$$
\begin{aligned}
& Z(t)=\sum_{n \leqslant \tau\left(x_{r}\right)} \frac{2}{\sqrt{n}} \cos \left\{t \omega_{n}\left(x_{r}\right)+\psi\left(x_{r}\right)\right\}+\mathcal{O}\left(x_{r}^{-1 / 4}\right), \\
& \tau\left(x_{r}\right)=\sqrt{\frac{x_{r}}{2 \pi}}, \quad t \in\left[x_{r}, x_{r}+V\right], \quad V \in\left(0, x_{r}^{1 / 4}\right),
\end{aligned}
$$

и аналогично для $x_{r} \rightarrow y_{r}$, где

$$
T_{0}<2 \pi L<x_{r}, y_{r}
$$

(см. $[5 ;(6.1)]$, см. $[6 ;(4.4)$ и замечание 6$])$.

ЗАмечАНИЕ 13. Назовем выражения

$$
\frac{2}{\sqrt{n}} \cos \left\{t \omega_{n}\left(x_{r}\right)+\psi\left(x_{r}\right)\right\}, \quad \cdots
$$

осцилляторами Римана, у которых:

(a) амплитуда

$$
\frac{2}{\sqrt{n}}
$$

(b) некогерентная локальная фазовая константа

$$
\psi\left(x_{r}\right)=-\frac{x_{r}}{2}-\frac{\pi}{8}
$$

(c) несинхронизированное локальное время

$$
t=t\left(x_{r}\right) \in\left[x_{r}, x_{r}+V\right]
$$

(d) локальный спектр циклических частот

$$
\left\{\omega_{n}\left(x_{r}\right)\right\}_{n \leqslant \tau\left(x_{r}\right)}, \quad \omega_{n}\left(x_{r}\right)=\ln \frac{\tau\left(x_{r}\right)}{n}, \quad \ldots .
$$

Замечание 14 . Теперь мы видим, что $Q$-система, у которой

$$
\left|\zeta\left(\frac{1}{2}+i t\right)\right|=|Z(t)|,
$$

действительно сложная колебательная система (см. (15), (31), (33)). 
Таким образом, получаем следующее (см. (15), (26), (31), замечание 14 и (33)).

СлЕДСТВИЕ 1. Набор метаморфоз

$$
\begin{aligned}
\prod_{r=1}^{k} \mid & \frac{\sum_{n \leqslant \tau\left(\alpha_{r}^{4}\right)}(2 / \sqrt{n}) \cos \left\{\alpha_{r}^{4} \omega_{n}\left(\alpha_{r}^{4}\right)+\psi\left(\alpha_{r}^{4}\right)\right\}+R\left(\alpha_{r}^{4}\right)}{\sum_{n \leqslant \tau\left(\beta_{r}^{4}\right)}(2 / \sqrt{n}) \cos \left\{\beta_{r}^{4} \omega_{n}\left(\beta_{r}^{4}\right)+\psi\left(\beta_{r}^{4}\right)\right\}+R\left(\beta_{r}^{4}\right)} \mid \\
& \sim \frac{\operatorname{arctg}(\sqrt{(a-b) /(a+b)} \operatorname{tg}(U / 2))}{\sqrt{(a-b) /(a+b)}(U / 2)} \frac{a+b \cos \left(\alpha_{0}^{4}\right)}{a+b}, \quad L \rightarrow \infty,
\end{aligned}
$$

соответствует формуле факторизачии (26).

ЗАмЕчАНИЕ 15. По теореме из п. 3.1 существуют функции контроля (25) (схема Голема) множества метаморфоз (34) осциллирующей $Q$-системы (15) (см. также (31), (33)).

ЗАмечАние 16. Механизм метаморфоз имеет следующий вид. Пусть (см. (25) и [4; $(2.2)]$ )

$$
M_{k}^{3}=\left\{\alpha_{1}^{4}, \ldots, \alpha_{k}^{4}\right\}, \quad M_{k}^{4}=\left\{\beta_{1}^{4}, \ldots, \beta_{k}^{4}\right\},
$$

где (см. $[4 ;(2.12)])$

$$
M_{k}^{3} \subset M_{k}^{1} \subset\left(T_{0},+\infty\right)^{k}, \quad M_{k}^{4} \subset M_{k}^{2} \subset\left(T_{0},+\infty\right)^{k} .
$$

Теперь если после случайной выборки таких точек (ср. условия [4; $(2.2)])$ мы получим, что

$$
\left(x_{1}, \ldots, x_{k}\right)=\left(\alpha_{1}^{4}, \ldots, \alpha_{k}^{4}\right) \subset M_{k}^{3}, \quad\left(y_{1}, \ldots, y_{k}\right)=\left(\beta_{1}^{4}, \ldots, \beta_{k}^{4}\right) \subset M_{k}^{4}
$$

(см. (35), (36)), то в точках (37) $Q$-система (15) меняет свою старую форму (“куколку") на новую форму ("бабочку"), а последняя находится под контролем функции $\alpha_{0}^{4}$.

ЗАмечАниЕ 17. Теперь должно быть ясно, что множество метаморфоз осциллирующей $Q$-системы также принадлежит семейству аналогов функционального уравнения Римана на критической прямой.

\section{5. О декомпозиции результата метаморфоз (34) на три части: сигнал, шум и погрешность}

В этом разделе используется терминология из теории обработки сигналов.

5.1. Напомним (см. [4; $(4.11)])$, что

$$
\widetilde{Z}^{2}(t)=\frac{|\zeta(1 / 2+i t)|^{2}}{\omega(t)}, \quad \omega(t)=\left\{1+\mathcal{O}\left(\frac{\ln \ln t}{\ln t}\right)\right\} \ln t .
$$

Поскольку в нашем случае

$$
t \rightarrow 2 \pi L
$$

то (см. $[4 ;(4.11),(4.12)])$

$$
\widetilde{Z}^{2}\left(\alpha_{r}^{4}\right)=\frac{\left|\zeta\left(1 / 2+i \alpha_{r}^{4}\right)\right|^{2}}{\{1+\mathcal{O}(\ln \ln L / \ln L)\} \ln t},
$$

ЗАмЕчАНИЕ 18. Основной вид асимптотической формулы для метаморфоз (34) - это

$$
\begin{aligned}
& \prod_{r=1}^{k}\left|\frac{\sum_{n \leqslant \tau\left(\alpha_{r}^{4}\right)}(2 / \sqrt{n}) \cos \left\{\alpha_{r}^{4} \omega_{n}\left(\alpha_{r}^{4}\right)+\psi\left(\alpha_{r}^{4}\right)\right\}+R\left(\alpha_{r}^{4}\right)}{\sum_{n \leqslant \tau\left(\beta_{r}^{4}\right)}(2 / \sqrt{n}) \cos \left\{\beta_{r}^{4} \omega_{n}\left(\beta_{r}^{4}\right)+\psi\left(\beta_{r}^{4}\right)\right\}+R\left(\beta_{r}^{4}\right)}\right| \\
& \quad \sim\left\{1+\mathcal{O}\left(\frac{\ln \ln L}{\ln L}\right)\right\} \frac{\operatorname{arctg}(\sqrt{(a-b) /(a+b)} \operatorname{tg}(U / 2))}{\sqrt{(a-b) /(a+b)}(U / 2)} \frac{a+b \cos \left(\alpha_{0}^{4}\right)}{a+b} .
\end{aligned}
$$


Так как последние два сомножителя в правой части формулы (38) - ограниченные функции для всех

$$
U \in(0, \pi), \quad L>\frac{1}{2 \pi} T_{0}
$$

(для всех фиксированных допустимых $k, a, b$, см. (22), (25)), то из (38) мы получаем следующее утверждение.

СлЕДСТВиЕ 2. Имеем

$$
\begin{aligned}
& \prod_{r=1}^{k}\left|\frac{\sum_{n \leqslant \tau\left(\alpha_{r}^{4}\right)}(2 / \sqrt{n}) \cos \left\{\alpha_{r}^{4} \omega_{n}\left(\alpha_{r}^{4}\right)+\psi\left(\alpha_{r}^{4}\right)\right\}+R\left(\alpha_{r}^{4}\right)}{\sum_{n \leqslant \tau\left(\beta_{r}^{4}\right)}(2 / \sqrt{n}) \cos \left\{\beta_{r}^{4} \omega_{n}\left(\beta_{r}^{4}\right)+\psi\left(\beta_{r}^{4}\right)\right\}+R\left(\beta_{r}^{4}\right)}\right| \\
& =\frac{a}{a+b} \frac{\operatorname{arctg}(\sqrt{(a-b) /(a+b)} \operatorname{tg}(U / 2))}{\sqrt{(a-b) /(a+b)}(U / 2)} \\
& \quad+\frac{b}{a+b} \frac{\operatorname{arctg}(\sqrt{(a-b) /(a+b)} \operatorname{tg}(U / 2))}{\sqrt{(a-b) /(a+b)}(U / 2)} \cos \left(\alpha_{0}^{4}\right)+\mathcal{O}\left(\frac{\ln \ln L}{\ln L}\right), \quad L \rightarrow \infty .
\end{aligned}
$$

5.2. Напомним (см. (25)), что

$$
\alpha_{0}^{4}=\alpha_{0}^{4}(L, U, k ; a, b)=\alpha_{0}^{4}(L, U)
$$

для допустимых фиксированных $k, a, b$.

(a) Мы видим, что первая функция в правой части (39) есть $L$-й член

$$
\begin{gathered}
f(2 \pi L+U)=g_{L}(U)=\frac{a}{a+b} \frac{\operatorname{arctg}(\sqrt{(a-b) /(a+b)} \operatorname{tg}(U / 2))}{\sqrt{(a-b) /(a+b)}(U / 2)}, \quad U \in(0, \pi), \\
g_{L}(U)=g_{L^{\prime}}(U), \quad L, L^{\prime}>\frac{1}{2 \pi} T, \quad 2 \pi L+U \in[2 \pi L, 2 \pi L+\pi),
\end{gathered}
$$

стационарной последовательности

$$
\left\{g_{L}(U)\right\}_{L>T_{0} /(2 \pi)}, \quad U \in(0, \pi) .
$$

Соответствующий сигнал определяется посредством (41), (42). Отсюда получаем, что определенный сигнал выражается через функции в правой части формулы (39) (см. (42)).

(b) Основным множителем второго члена является следующая функция:

$$
\cos \left(\alpha_{0}^{4}\right), \quad \alpha_{0}^{4} \in \alpha_{0}^{4}(L, U),
$$

где (из (14), (40))

$$
\alpha_{0}^{4}=\varphi_{1}(d) \in(2 \pi L, 2 \pi L+U), \quad d=d(L, U),
$$

и $\varphi_{1}(d)$ - значение лестницы Иакова. То есть распределение значений

$$
\alpha_{0}^{4} \in(2 \pi L, 2 \pi L+U), \quad U \in(0, \pi),
$$

как можно предположить, очень сложное.

Следовательно, вторую функцию будем характеризовать как шум - неполезную часть сигнала. Шум можно регулировать путем изменения параметра $b$,

$$
a>|b|
$$

(см. (22)), т.е. путем уменьшения $|b|$.

(с) Третью функцию будем называть (мелкой) погрешностью, так как

$$
\mathcal{O}\left(\frac{\ln \ln L}{\ln L}\right) \stackrel{L \rightarrow \infty}{\longrightarrow} 0 .
$$


ЗАмЕчАниЕ 19. Конечное состояние метаморфоз в (39) разбивается на три части: сигнал, шум и погрешность.

\section{6. Множество различных метаморфоз в (34)}

Разумеется, существует точка $U_{0} \in(0, \pi)$ такая, что (см. (41)

$$
\left.g_{L}^{\prime}(U)\right|_{U=U_{0}} \neq 0 \quad \text { для всех } L>\frac{T_{0}}{2 \pi},
$$

т.e.

$$
\begin{gathered}
g_{L}^{\prime}(U) \neq 0, \quad U \in O_{\delta}\left(U_{0}\right)=\left(U_{0}-\delta, U_{0}+\delta\right), \\
U^{\prime} \neq U^{\prime \prime} \Longrightarrow g_{L}\left(U^{\prime}\right) \neq g_{L}\left(U^{\prime \prime}\right) \quad \text { для всех } \quad U^{\prime}, U^{\prime \prime} \in O_{\delta}\left(U_{0}\right)
\end{gathered}
$$

для подходящего $\delta>0$.

Далее, мы будем предполагать, что существуют такие

$$
U_{1}, U_{2} \in O_{\delta}\left(U_{0}\right), \quad U_{1} \neq U_{2},
$$

чTо

$$
\begin{aligned}
\alpha_{r}^{4}\left(U_{1}, L\right) & =\alpha_{r}^{4}\left(U_{2}, L\right), & r & =0,1, \ldots, k, \\
\beta_{r}^{4}\left(U_{1}, L\right) & =\beta_{r}^{4}\left(U_{2}, L\right), & r & =1, \ldots, k,
\end{aligned}
$$

для всех

$$
L>\widetilde{L}>\frac{T_{0}}{2 \pi} .
$$

В этом случае путем сравнения формул (38) для $U_{1}, U_{2}$ мы получаем, что

$$
\frac{\operatorname{arctg}\left(\sqrt{(a-b) /(a+b)} \operatorname{tg}\left(U_{1} / 2\right)\right)}{\sqrt{(a-b) /(a+b)}\left(U_{1} / 2\right)}=\left\{1+\mathcal{O}\left(\frac{\ln \ln L}{\ln L}\right)\right\} \frac{\operatorname{arctg}\left(\sqrt{(a-b) /(a+b)} \operatorname{tg}\left(U_{2} / 2\right)\right)}{\sqrt{(a-b) /(a+b)}\left(U_{2} / 2\right)},
$$

т.е. в предельном случае имеем равенство

$$
\frac{\operatorname{arctg}\left(\sqrt{(a-b) /(a+b)} \operatorname{tg}\left(U_{1} / 2\right)\right)}{\sqrt{(a-b) /(a+b)}\left(U_{1} / 2\right)}=\frac{\operatorname{arctg}\left(\sqrt{(a-b) /(a+b)} \operatorname{tg}\left(U_{2} / 2\right)\right)}{\sqrt{(a-b) /(a+b)}\left(U_{2} / 2\right)},
$$

которое противоречит (43).

Следовательно, для любых

$$
U^{\prime}, U^{\prime \prime} \in O_{\delta}\left(U_{0}\right), \quad U^{\prime} \neq U^{\prime \prime}
$$

существует бесконечная подпоследовательность

$$
\{\bar{L}\} \subset\{L\}, \quad \bar{L}>\widetilde{L},
$$

такая, что (см. (44))

$$
\begin{aligned}
& \left(\alpha_{0}^{4}\left(U^{\prime}, \bar{L}\right), \alpha_{1}^{4}\left(U^{\prime}, \bar{L}\right), \ldots, \alpha_{k}^{4}\left(U^{\prime}, \bar{L}\right), \beta_{1}^{4}\left(U^{\prime}, \bar{L}\right), \ldots, \beta_{k}^{4}\left(U^{\prime}, \bar{L}\right)\right) \\
& \quad \neq\left(\alpha_{0}^{4}\left(U^{\prime \prime}, \bar{L}\right), \alpha_{1}^{4}\left(U^{\prime \prime}, \bar{L}\right), \ldots, \alpha_{k}^{4}\left(U^{\prime \prime}, \bar{L}\right), \beta_{1}^{4}\left(U^{\prime \prime}, \bar{L}\right), \ldots, \beta_{k}^{4}\left(U^{\prime \prime}, \bar{L}\right)\right) \quad \text { для всех } \quad \bar{L} \in\{\bar{L}\} .
\end{aligned}
$$

Поэтому, из (45) получаем

СЛЕДСТВИЕ 3. Существует бесконечное множество различных метаморфоз (34).

Я благодарю Екатерину Карацубу и Михала Деметриана за моральную поддержку моего исследования лестниц Иакова. 


\section{Список литературы}

[1] G. H. Hardy, Divergent Series, Oxford, Oxford Univ. Press, 1949.

[2] E. C. Titchmarsh, The Theory of the Riemann Zeta-Function, Clarendon Press, Oxford, 1951.

[3] E. Landau, Handbuch der Lehre von der Verteilung der Primzahlen, Teubner, Leipzig, 1909.

[4] J. Moser, Jacob's Ladders, Riemann's Oscillators, Quotient of the Oscillating Multiforms and Set of Metamorphoses of This System, arXiv: 1506.00442.

[5] J. Moser, Jacob's Ladders, $\zeta$-Factorization and Infinite Set of Metamorphosis of a Multiform, arXiv: 1501.07705 .

[6] J. Moser, Jacob's Ladders, New Properties of the Function $\arg \zeta(1 / 2+i t)$ and Corresponding Metamorphoses, arXiv: 1506.07967.

[7] Я. Мозер, "Лестницы Якова и почти точные асимптотические представления интеграла Харди-Литтлвуда", Матем. заметки, 88:3 (2010), 446-455.

[8] J. Moser, "Jacob's ladders, the structure of the Hardy-Littlewood integral and some new class of nonlinear integral equations", Теория чисел, алгебра и анализ, Сборник статей. К 75-летию со дня рождения профессора Анатолия Алексеевича Карацубы, Тр. МИАН, 276, МАИК, М., 2012, 213-226.

[9] L. Euleri, Institutionum calculi integralis, Volumen primum, Impensis Academiae Imperialis Scientiarum, Petropoli, 1768; Л. Эйлер, Интегральное исчисление, Гостехиздат, М., 1956. 